\title{
EDITORIAL
}

\section{Systems dynamics of biology}

\author{
David Lloyd \\ Microbiology Group, Cardiff School of Biosciences, Cardiff University, Cardiff, Wales, U.K.
}

Received: $22^{\text {nd }}$ March 2005

Published: $30^{\text {th }}$ March 2005

\begin{abstract}
Summary
As more information accumulates on the detailed dynamics of life processes, emphasis on steady state behaviour gives way to the recognition that the organism is a multi-oscillator. Systems biology takes account of the controls and balances on inputs and outputs between the environment and the open system of the functioning organism. The matching of the system to its periodic environment performed by biological clocks (circadian, tidal, lunar, annual) is belatedly acknowledged and exploited in applied biomedicine, even as detailed mechanisms continue to be elucidated. Ancestral shorter-period (ultradian) oscillations, rhythms and clocks are all-pervasive in intracellular regulation and control. Yeast in continuous culture shows all these characteristics as well as population coherence. Temporal organisation of tissues, organs and of the whole organism; its construction, operation and maintenance are extensions of these dynamic principles.
\end{abstract}

Keywords: circadian rhythms - ultradian rhythms - cell and tissue dynamics

"Life is what is lost in the test-tube." Chargraff 1978

\section{INTRODUCTION}

The enormous success of reductionism in biology, beginning with the extraction of active enzymes from yeast using extreme forces (Buchner 1898)

David Lloyd, Microbiology Group, Cardiff School of Biosciences, Cardiff University, P O Box 915, Cardiff CF10 3TL, Wales, U.K.

1loydd@Cardiff.ac.uk have obscured the real object of study. Although physiologists have never veered from the importance of in vivo measurement, the power of molecular biology and the emphasis on structure has relegated dynamic studies to a secondary activity. As we bank information (genomics, protein structures and metabolic data) with accelerating scope and confidence, it is time to consider again how to integrate the accumulated detail into the wisdom that can only stem from an understanding of the whole. What we still need to know are the fundamental rules that govern the amazing systems involved and the complicated 
emergent behaviour that follows as a consequence. Here, I review some of the recently available basic systems dynamics that underlie the complexities and coherence of the whole organism.

\section{SYSTEMS BIOLOGY}

Systems biology, a recent "new" initiative (Kitano 2002, Anon 2004) echoes ideas dating back to antiquity. Trying to encapsulate the special properties of living matter, Heraclitus made an analogy with the flow of a stream: "You can never step into the same river twice, as fresh water is always flowing towards you." Norbert Weiner too, conveyed the evanescence of the living state: "It is the pattern maintained by this homeostasis, which is the touchstone of our personal identity. Our tissues change as we live: the food we eat and the air we breathe become flesh of our flesh and bone of our bone, and the momentary elements of our flesh and bone pass out of our body every day with our excreta. We are but whirlpools in a river of ever-flowing water. We are not the stuff that abides, but patterns that perpetuate themselves".(Weiner 1954)

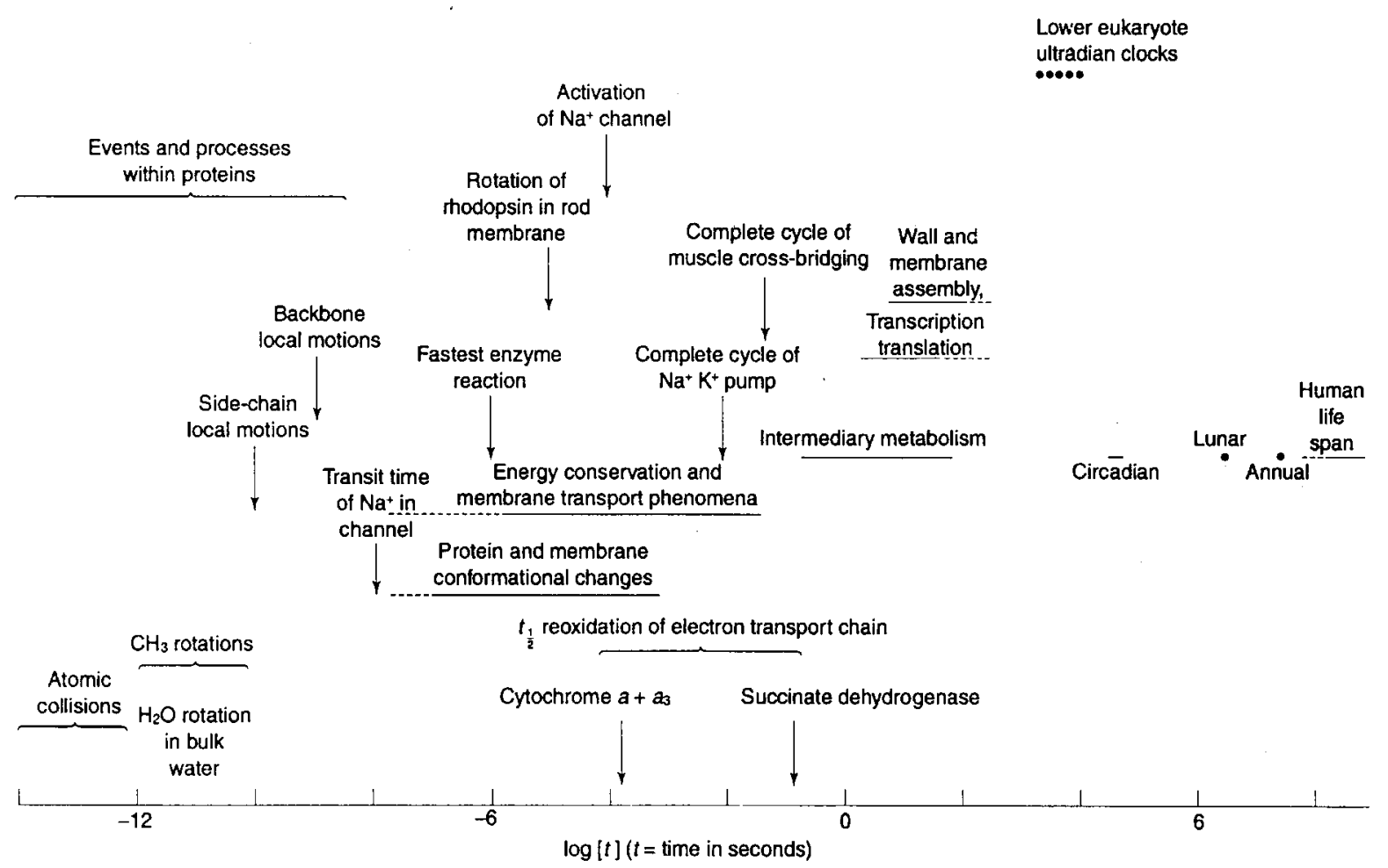

Fig. 1. The time domains of living systems. Approximate relaxation times (to a return of $1 / \mathrm{e}$ of a small perturbative effect) are indicated.

With the growing realization of the pervasive rhythmicity of physiological and biochemical functions, especially on the circadian time scale $(\tau \simeq 24 \mathrm{~h})$, the serious consequences of deranged temporal organisation (e.g. in shift workers, jet lag and certain depressive states) became apparent.

The appreciation of the medical importance of disturbed dynamic states at a biochemical level dates back at least to the 1970's, when Gilbert (1974) suggested that perturbation of the cell division cycle could be modelled using the dynamic systems approach; more recently the same investigator provided new insights into the loss of autodynamic control during tumour development (Gilbert 1984), and also during the aging process
(Gilbert 1995). A further attempt to more closely integrate phenotype with the genome and the humeral system dates back more than a decade (Lloyd and Rossi 1993). A quickening interest in the importance of 'Global systems biology' currently arises from a gradual realization that understanding of the interaction of the human genome with those of parasites and of diverse symbiotic gut microbes as well as with the environment, is essential for the prediction of diseases and the effectiveness of drug administration (Nicholson et al. 2004). An integrated view of human physiological performance in health and sickness will provide more subtle treatment protocols than those now 
available. Other far-reaching implications of systems biology encompass the search for new drugs and for new strains of microbes for maximal product formation (Cortassa et al. 2002).

Yet a universally-agreed definition of systems biology is hard to find. To most it suggests an initial microarray-based genome-wide transcriptome analysis, then proteomics (2D separation of thousands of proteins on gels followed by mass spectrometric sequencing of proteolytic-enzyme generated peptides) and metabolomics (analysis, identification and quantification of all the hundreds of metabolic pools of a cell type). Finally, metabolic control analysis may simplify understanding of regulatory principles that underlie distributed control in metabolic pathways. Subsequent steps would require synthesis of this information to provide a new insight into the functioning of the whole sub-system, and then use of mathematical modelling to identify important state variables. Clearly this approach requires massive investment in specialist techniques. The magnitude of the enterprise is formidable, especially where human biology is concerned. For the microbial workhorses that generate many of our new biologically-active compounds (antibiotics, psychotic drugs, antihypertensive agents, immunosuppressant agents, anti-retroviral therapeutics, cytotoxic anti-cancer drugs, etc.), where we are asking questions of single-celled organisms, filamentous fungi, or plants the task is much simpler, but still not straight forward.

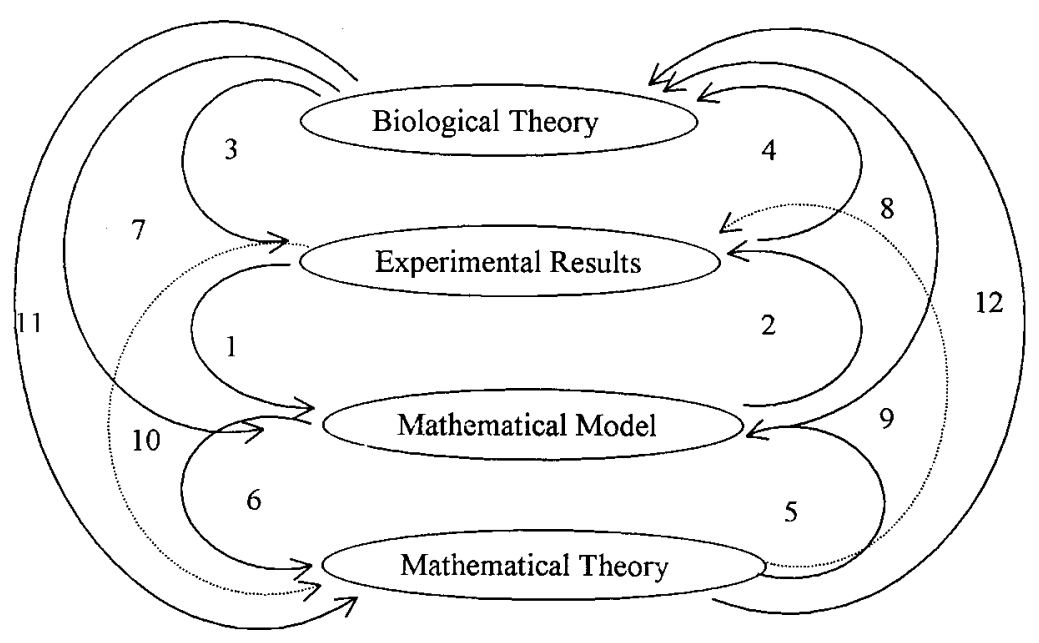

Fig. 2. The iterative interations involved in theoretical biology (Fuentes-Pardo et al. 2003)

The time-structures of biological process in all its complexity (Fig. 1) indicates that static snapshots of the living machinery are not the last word. Indeed, just as yesterday's proteome is not the same as today's (Beynon 2005), this morning's is not the same as this evening's. For the metabolic pools, a moment-to-moment description is necessary to document their oscillatory states (Brodsky 1975, 2005, Roussel and Lloyd, under preparation). The time-structure of life encompasses many decades, when measured in seconds and there may be a self-similarity of rhythmic and oscillatory processes (Aon et al. 2000).

\section{TIME SCALES}

"What are called structures are slow processes of long duration, functions are quick processes of short duration" (von Bertalanffy 1952). This prescient statement emphasizes that we must not merely dissect the organism but be also cognisant of its time structure. Weber (1976) has pointed out the hazards of overlooking the relevance of working on the appropriate time-scale, e.g. when investigating conformational change in protein structure: This situation is analogous with that involved in deciding whether a mountain is a rigid or a flexible structure. Evidently over a period of a human lifetime, of the order of tens of years, the mountain would not change appreciably its shape, 
but this conclusion could not be extended as valid over periods of geological time, of the order of $10^{8}$ years. The latter time interval would correspond to $1 \mathrm{~s}$ on the molecular time scale if the year is 1 ns. We must therefore guard against deriving rigorous conclusions as the "molecular rigidity" from observations on the nano-second time scale".

Systems biology attempts to understand the dynamics of change during the life of the organism from its independent origin to its ultimate demise (Lloyd et al. 1984, Lloyd 1998). An important aid to separation of the paramount from the marginalia along this trajectory is the elaboration and application of mathematical models. Interactive iteration (Fig. 2) between the experimental investigator and the mathematician is necessary to produce a useful representation not too divorced from reality (Fuentes-Pardo et al. 2003).

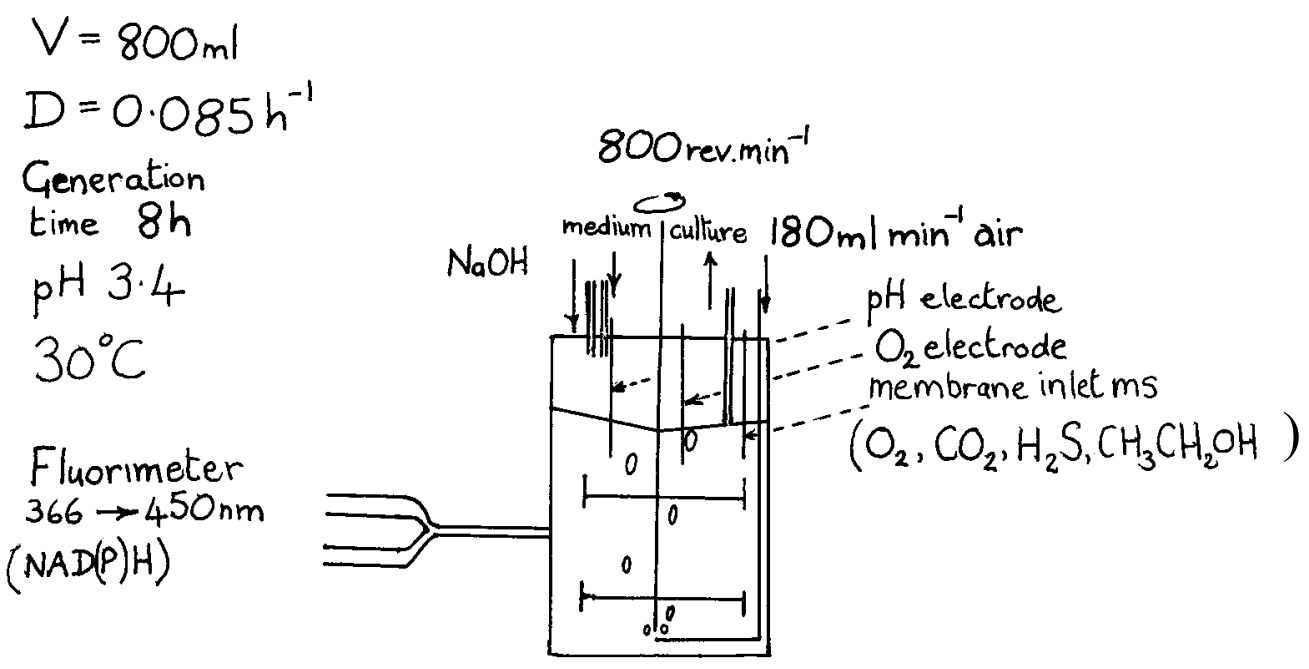

Fig. 3. The continuous culture of yeast

\section{MODELS OF THE CELL DIVISION CYCLE}

Modelling of the cell division cycle has been repeatedly revisited in recent years. Selkov (1970) produced a model based on dithiol-disulphide cycles, drawing on the experiments of Mano (1968), who had shown the importance of a thiol/disulphide cycle and the changing redox state of the sea urchin eggs during early embryogenesis. Other emphasis was placed on phosphorylation cycles in the proposals of Gilbert (1974). Chernavskii et al. (1977) considered oxidation of membrane lipids to free radical species and provided a basic framework for a system of equations. These were developed more recently (Lloyd and Volkov 1990) to account for the quantized cell cycle times, first observed for mammalian cells in culture by Klevecz (1976). Over certain restricted values for state variables, chaotic operation was predicted (Mackey 1986, Lloyd et al. 1992) and this conclusion led to a suggestion that a controlled chaotic attractor (Lloyd and Lloyd 1993) might be responsible for the multi-oscillator outputs experimentally observable in biological systems (Klevecz 1993).

More detailed models of cell division cycle controls e.g. by Tyson et al. (2003) take into account detailed information on signalling pathways. Others have built mathematical models of calcium oscillations (DuPont et al. 2003), and of circadian rhythms (Gonze et al. 2004). Temperature compensation of biological clocks has been dealt with by Ruoff et al. (2000). These representations take into account much of the experimental data that is to hand at the time of their formulation.

One disadvantage of such detailed expositions is that a new discovery can at a stroke negate their value.

Progressive refinement can seem in hindsight rather trivial by comparison with radical new understanding, which inevitably comes from the application of new experimental techniques to old 
problems: one good experiment is worth a thousand models. Nevertheless, as an aid to critical analysis of complex systems, models have played an important part; their predictive utility cannot be disputed and is often non-intuitive.

\section{EXPERIMENTAL SYSTEM - YEAST IN CONTINUOUS CULTURE}

As an example of where systems biology can be studied to advantage, the yeast Saccharomyces cerevisiae has many attractions. Under optimum culture conditions it can reproduce (bud) every 90 min: during this cell division cycle time it has to approximately double its biomass and partition the newly-synthesized materials between mother cell and bud. For eukaryotes, its genome was the first to be sequenced (in 1996).

In a continuous culture (Fig. 3) its reproductive cycle time is controlled by the dilution rate of its growth medium. The system is open for liquid and gas flow, and culture can be continued for extended periods of time (up to years). In the continuous culture vessel, temperature, $\mathrm{pH}$, stirring rate, aeration rate and nutrient supply are strictly controlled. Residual dissolved $\mathrm{O}_{2}$ is monitored continuously, and as the supply rate does not vary, any changes in $\mathrm{O}_{2}$ consumption are reflected in a reciprocal change in dissolved $\mathrm{O}_{2}$ levels, i.e., we are measuring the respiratory activity of the organisms (Satroutdinov et al. 1992). Initially cells are grown in a batch culture, where near the onset of nutrient and stored energy reserve depletion, the dissolved $\mathrm{O}_{2}$ begins to oscillate. Continuous growth is initiated after all the carbon sources have been exhausted by starting the culture medium flow. The immersed $\mathrm{O}_{2}$ electrode provides a stable device for continuous measurement. The output (Fig. 4) is not sinusoidal, but rather has a distinct wave form, its trough-to-peak amplitude is about half that of the highest levels attained, and its period abut $40 \mathrm{~min}$. Experimental changes of temperature through a series of discrete steps (Murray et al. 2001) reveal its clock-like characteristic, compensation of period length (Fig. 4).

Other characteristics suggest that highlyconserved signalling pathways are involved, including dose-dependent prolongation of period length by $\mathrm{Li}^{+}$(Fig. 5), and perturbation of waveform and period by A-type monoamine oxidase inhibitors such as phenelizine (Salgado et al. 2002). It is remarkable that yeast and brain function have common features with respect to higher-order properties such as rhythmicity. One of the most interesting facets of this oscillating yeast culture is the mechanism that underlies coherent behaviour. If each organism was performing all its functions independently, then a steady state would result in which time averaging across the population would give a time-independent output. In fact the individuals (more than $10^{9}$ of them) have become synchronised; that the oscillation of dissolved $\mathrm{O}_{2}$ is of such high amplitude indicates that synchronisation must have involved a majority of the individual organisms. Essential to the respiratory synchrony exhibited is the pulsatile release of $\mathrm{H}_{2} \mathrm{~S}$ (Sohn et al. 2000) (a potent inhibitor of cytochrome $c$ oxidase, the terminal component of the mitochondrial respiratory chain). This volatile small molecule is rapidly oxidized by $\mathrm{O}_{2}$ dissolved in the culture and so shows a series of spikes at $40 \mathrm{~min}$ intervals (Fig. 6). Note too that it has recently been shown that $\mathrm{H}_{2} \mathrm{~S}$ spikes are an important feature of neurotransmission in brain function (Kimura 2002). Another transiently produced and rapidly-eliminated volatile messenger substance responsible for extracellular communication between cells of the culture is acetaldehyde, a fermentation product of the yeast (Fig. 6). The importance of both these compounds, and possibly that of other related easily diffusible chemical species has been shown by their phaseshifting capabilities when added to the culture at certain times during the $40 \mathrm{~min}$ cycles (Murray et al. 2003).

Other redox active components also show $40 \mathrm{~min}$ cycles. Although its waveform is more complex, NAD $(\mathrm{P}) \mathrm{H}$ also shows a clear phase relationship with that of dissolved $\mathrm{O}_{2}$, as does reduced glutathione GSH, (Fig. 7) (Murray et al. 1999). Therefore the observed respiratory cycles are characterized by redox switching. Evidence for this also comes from the perturbative effects of 5nitro-2-furaldehyde (a GSH synthesis inhibitor) and DL-butathionene $(\mathrm{S}, \mathrm{R})$-sulphoximine (a $\gamma$-glutamyl cysteine inhibitor). Furthermore, additions of GSH and GSSG both perturb the respiratory oscillation. Nitrosonium anions $\left(\mathrm{NO}^{+}\right.$, eg. from $\mathrm{Na}$ nitroprusside are also perturbants; their preferred targets are thiols and metalloproteins (Murray et al. 1998).

Mitochondrial energization is responsible for the accelerated respiratory rates observed in the continuous cultures when dissolved $\mathrm{O}_{2}$ is at a minimum (Lloyd et al. 2002). Thus during the $40 \mathrm{~min}$ cycle, the changes involved are like those seen with isolated mitochondria when they already have excess respiratory substrates but become fully activated only when further supplemented by added ADP. Massive changes in ionic balances across the inner mitochondrial membrane accompany this transition; its reversal to restore the initial state occurs on completion of ADP phosphorylation. Changes occur in cytochome redox states, inner mitochondrial membrane potential (as measured by fluorometric assay using Rhodamine 123, cationic dye internalised by mitochondria), and 
conformational states (as revealed in electron micrographs of sectioned fixed yeasts).

Confirmation that energy conservation within mitochondria is closely linked to the changing rate of respiration responsible for the respiratory oscillation in the continuous cultures came from studies on the effects of addition of uncouplers of mitochondrial energy-yielding reactions (protonophores). Immediate acceleration of respiration leads to loss of the $40 \mathrm{~min}$ respiratory cycle and when the clock output becomes fully detectable again (about 10 cycles to regain full amplitude) a further effect was observed. A slower rhythm (period $8 \mathrm{~h}$ ) was seen as an envelope superimposed upon the clock cycle (Fig. 8). At the dilution rate employed $\left(0.085 \mathrm{~h}^{-1}\right)$ this period corresponds to the cell division cycle time. All these in vivo responses are much slower than mitochondrial activities in vitro (time scales for reactive energy conservation and ionic balances are measured on a range ms-s): these organelles are enslaved within the cell to a slower dynamic (Lloyd and Edwards 1984). They march to a slower drum that plays elsewhere.

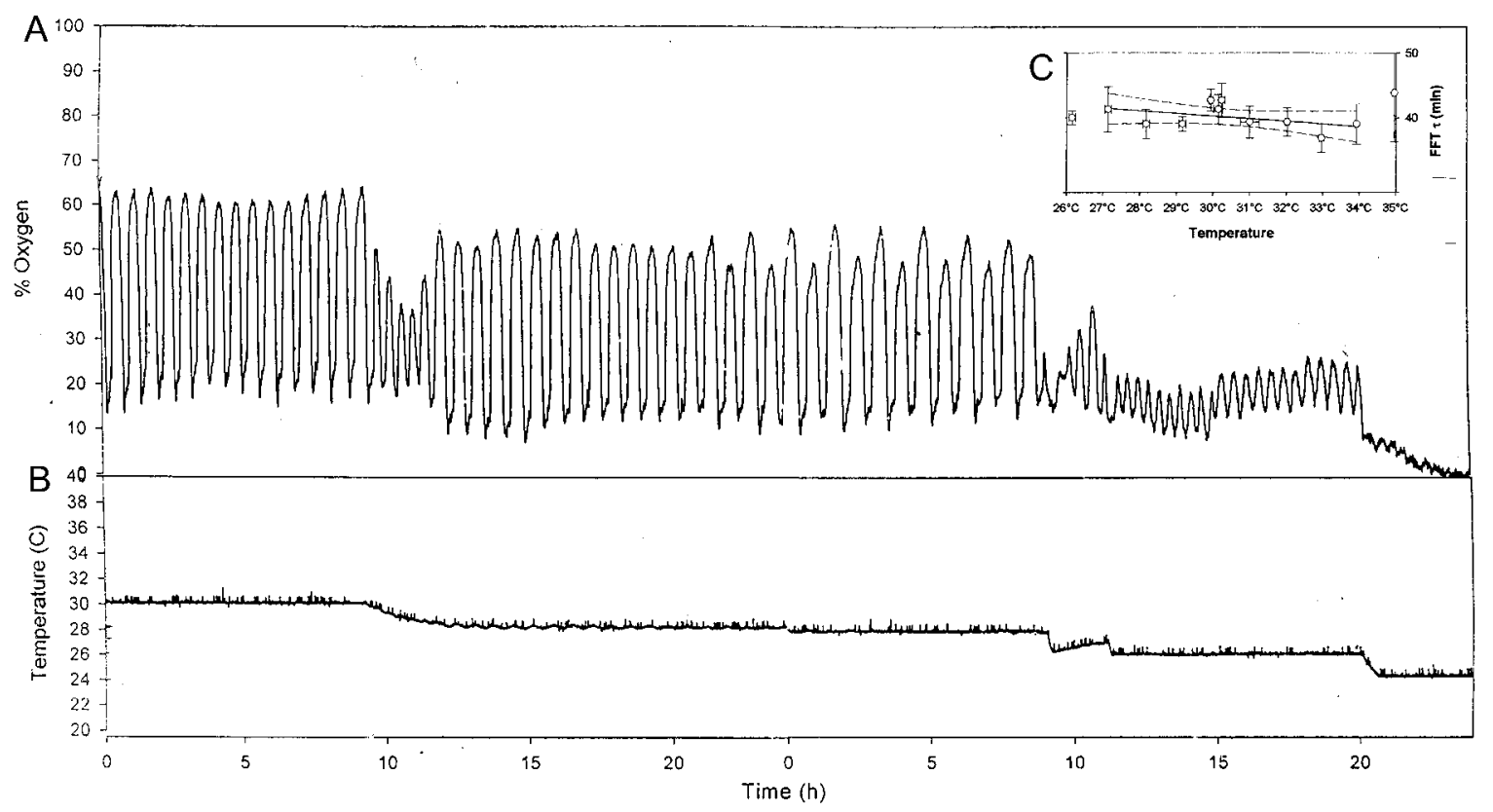

Fig. 4. Effects of stepwise alterations of temperature on the respiratory oscillation. (A) Dissolved $\mathrm{O}_{2}$, monitored by the submerged $\mathrm{O}_{2}$ electrode. (B) Temperature of the culture. (C) Temperature dependence of the period of the oscillation as indicated by fast Fourier transfer analysis (Murray et al. 2001).

Microarray analysis of mRNA species during respiratory oscillations transcriptome too is enslaved to the 40 min clock. Thus transcripts could be allocated into two classes; 650 were maximally expressed during active respiration whereas 4679 were observed maximally when cellular redox state was most reducing. Moreover, every 40 min cycle sees a subpopulation of cells gated in the $\mathrm{G}_{1}-\mathrm{S}$ transition. Most of the DNA replication and RNA synthesis is temporally partitioned into a period of the cell cycle when the intracellular state is most reduced.

We conclude that this global intracellular synchronization of different processes from transport of nutrients across the plasma membrane, through the whole metabolic network, and intracellular signalling, to the more highly organized organelle functions in mitochondria, the transcription of messenger RNA and DNA replication constitutes emergent coherence (Lloyd and Murray 2005) dictated by the ultradian $(\tau=$ $40 \mathrm{~min}$ ) clock. Were we able to employ more rapidly responding monitors then it seems likely that we would reveal a similar timeframe in a higher frequency domain: 2-photon excitation of individual yeast cells provides evidence for this (Lloyd et al. 2003). On a slower scale, there is good evidence for circadian timekeeping in yeast (Edmunds 1988). 

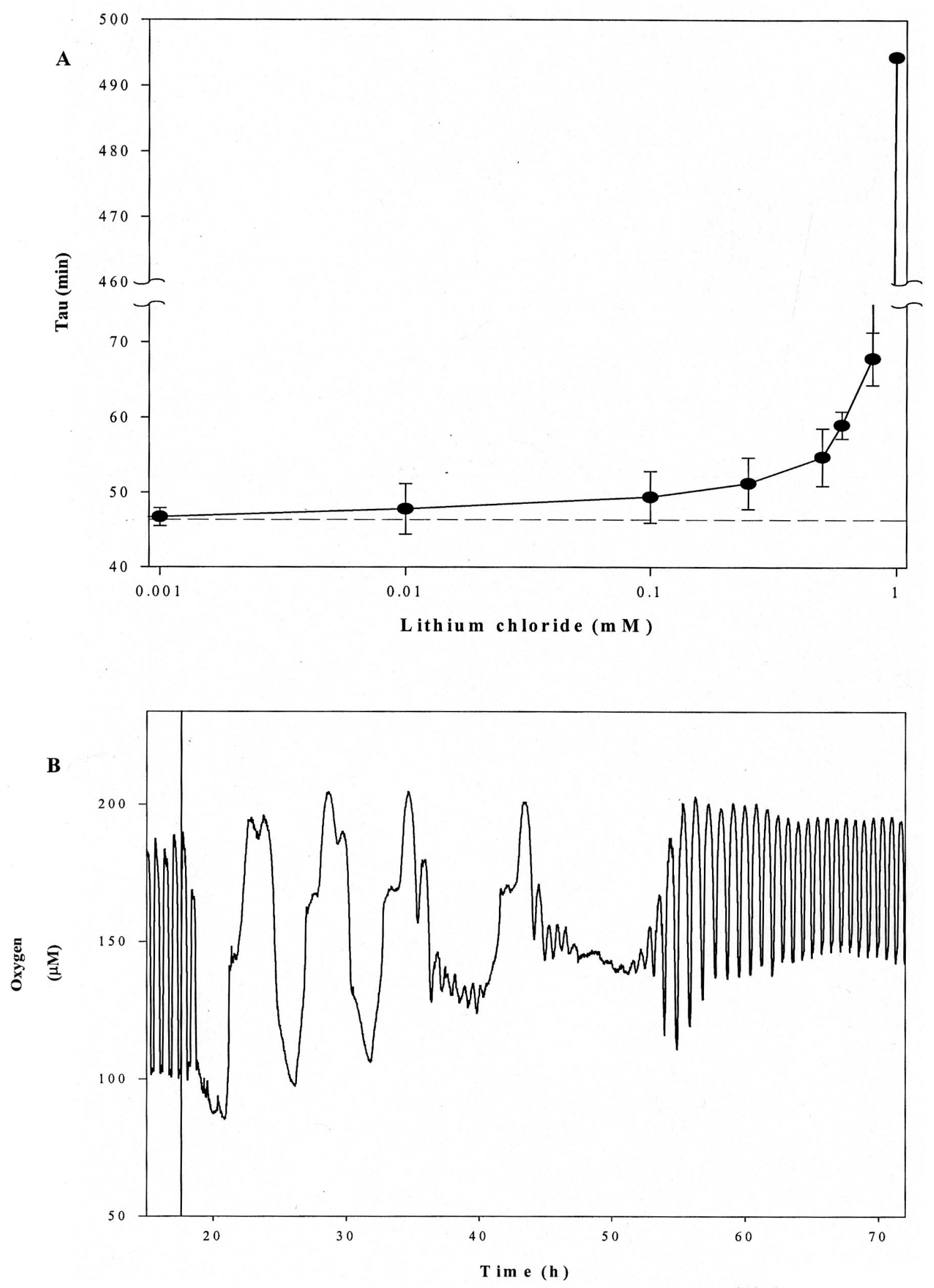

Fig. 5. Effects of $\mathbf{L i C l}$ additions on period of the respiratory oscillation. The horizontal line indicates periodicity of the untreated culture (Salgado et al. 2002). 


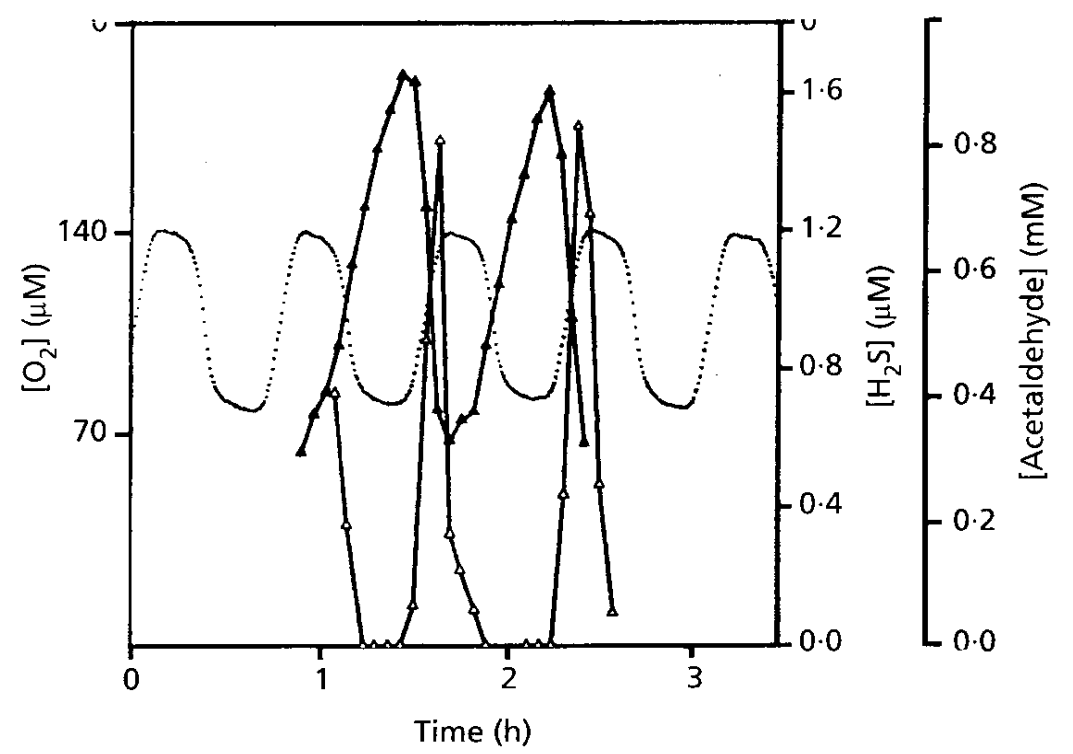

Fig. 6. Acetaldehyde (closed triangles) and $\mathbf{H}_{2} \mathbf{S}$ (open ones) production in the continuous culture (Murray et al. 2003)

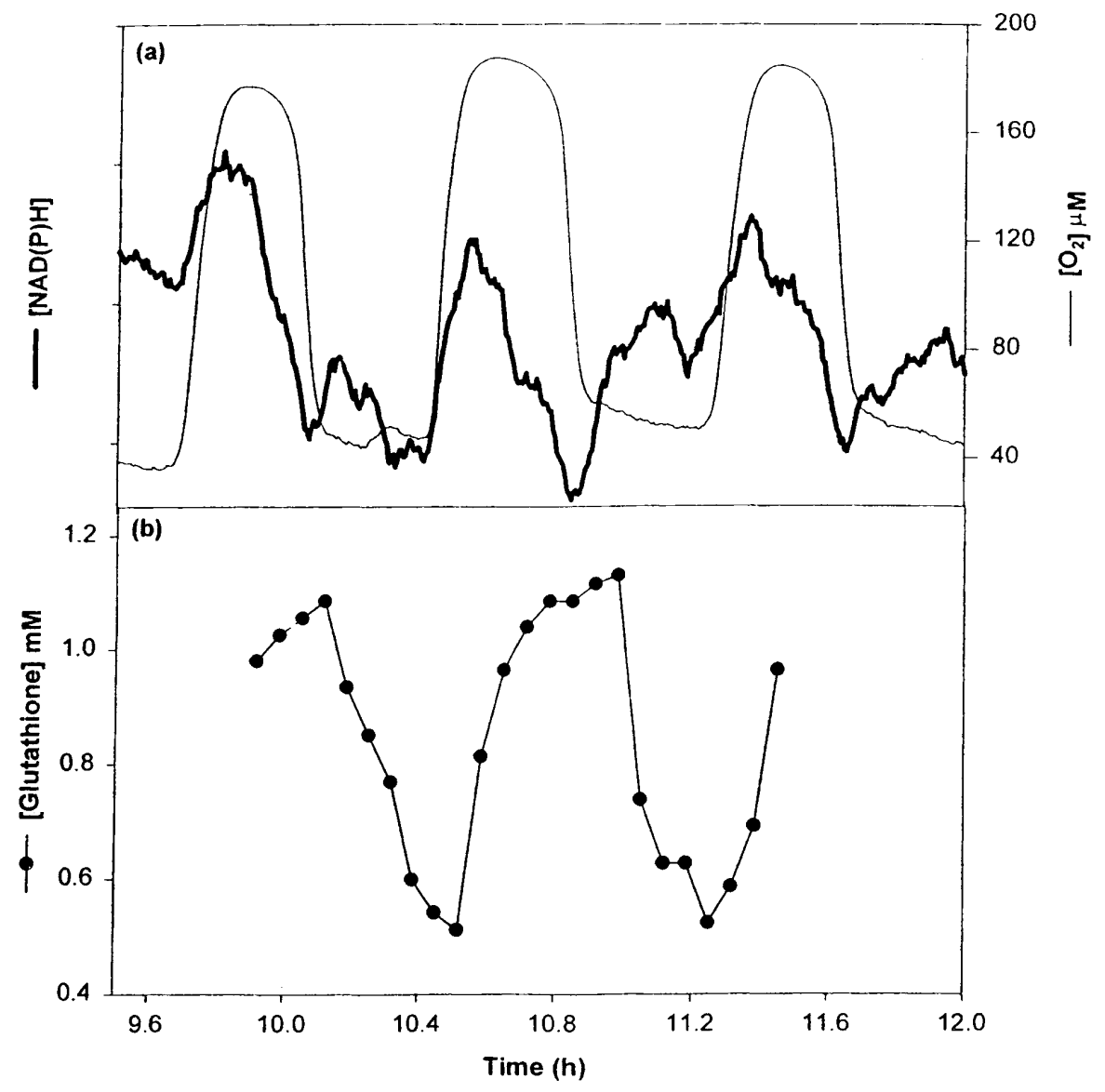

Fig. 7. Non-invasive monitoring of culture redox state by (a) direct fluorimetric $(366 \rightarrow 450 \mathrm{~nm})$ continuous measurement of NADH (heavy continuous line) alongside dissolved $\mathrm{O}_{2}$ (fine continuous line): (b) shows the glutathione content of organisms sampled from the culture (Murray et al. 1999). 


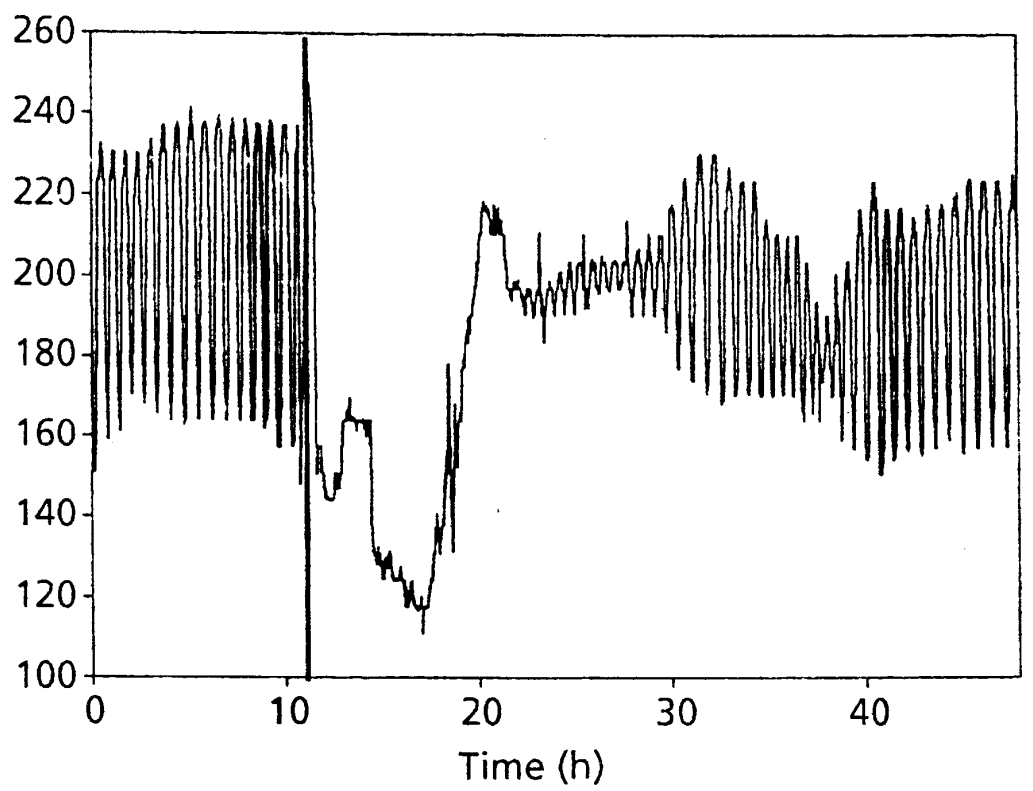

Fig. 8. Effects of addition of $5 \mu \mathrm{M}$ CCCP on continuous culture of $S$. cerevisiae previously spontaneously sychronised to the cell division cycle (Lloyd 2003)

Mathematical modelling of the 40 min ultradian clock has been shown to provide evidence that $\mathrm{SO}_{4}{ }^{2-}$ assimilation, ethanol degradation and respiration as key pathways that can give most of the characteristic kinetics of the system (Wolf et al. 2001, 2005). In a more recent model, Henson (2004) has extended this model to look at the intercellular communication across a population of $10^{4}$ individuals so as to simulate the population synchronization and thereby account for the oscillatory output of the culture. That the $40 \mathrm{~min}$ clock signal has outputs almost throughout the entire metabolic network and also entrains mitochondrial energetics the transcriptome as well as making the entire cellular population coherent is indicative of its fundamental importance. Clearly it cannot be explained or modelled on the basis of a simple limit cycle fashioned out of a small scale metabolic network. The robustness of the oscillation to external environmental variations (temperature, $\mathrm{pH}$, nutrient supplies) indicates a complex mechanism; a limit cycle would be more sensitive to physical and chemical perturbation (Wolf et al. 2005). The most likely explanation for this stubbornly-defended trajectory is that a massive array of coupled oscillators becomes selfsynchronized, as happens when a million fireflies flash in unison (Strogatz 2000).

\section{CONCLUSIONS}

The temporal organization of organisms, their tissues and cells is dominated by biological clocks that operate in and between distinct temporal domains and act as time-frames for convergence of parallel processing. The whole system constitutes a heterarchy where controls operate within and between the levels (Yates 1992). Their synchronizing outputs make systems biology a more tractable goal. Clearly the detailed dissection of metabolite pools, proteins, transcriptional and replication controls, and of the genome sequence, infers an enormous complexity, but underlying and overlying this complexity are coordinating influences that bring to the whole a simpler concerted dynamic (Lloyd and Murray 2005). By way of analogy, the system described for the yeast population outlined in the previous section shares many features with the human heart. In the latter case (Noble 2002, 2005) more than 40 years has seen progressive refinement of understanding of ion transport through membrane channels and their pivotal role in the excitability of cardiac muscle. Coherence of the contraction of geometrically arranged muscle fibres and bundles of muscles is an extremely highly ordered system: the integrated dynamics from ion channel conductance, through intercellular coordination and tissue coherence results in the emergence of the integrated mechanical functioning of the whole organ. Diseased states (tachycardia, ischemia, heart failure) can be understood on the basis of mathematical models. Mutations of $\mathrm{Na}^{+}$channel proteins can be related to abnormal electrocardiogram (ECG) outputs in congenital conditions.

In both systems an oscillation percolates not only throughout a cellular network, including 
organelles, transcriptome and the metabolome, but also the entire cell population. This oscillatory state is not a exceptional curiosity found only in a peculiar system, but rather a universal trait, necessary for the maintenance of a robust metabolic autodynamic state, characteristic of normally-healthy cells. In yeast, further consequences include the quantization of cell division cycle times (cell doubling times) as integral values of ultradian clock cycle times. As the latter have different values in different species, so will cell doubling times; this has been shown for ten different strains of $S$. cerevisiae, two other yeasts and seven protist species (Lloyd and Rossi 1993). The human ultradian clock has a cycle time of $90 \mathrm{~min}$. Aging of cells is widely held to be due, at least in part, to an accumulation of reactive $\mathrm{O}_{2}$ species-produced damage to cellular components, especially lipids and proteins. Oxidative stress varies during the mitochondrial energization cycles, leading to the intriguing theory that cell aging can be quantitatively measured in ultradian cycles (Lloyd et al. 2003).

\section{REFERENCES}

Anon A.: End of the interlude? Nature Biotechnol. 22: 1191-1200, 2004.

Aon M.A., Cortessa S., Lloyd D.: Chaotic dynamics and fractal space in biochemistry: simplicity underlies complexity. Cell Biol. Int. 24: 581-587, 2000.

Beynon J.R.: The proteome as a dynamic entity - is yesterday's proteome the same as today's? Biochem. Soc. Trans., abstract SA038, 2005.

Brodsky V.J.: Protein synthesis rhythm. J. Theor. Biol. 55:167-200, 1975.

Brodsky V.J.: Direct cell-cell communication. A new approach due to recent data on the nature and self organization of ultradian circahoralian intracellular rhythms. Biol. Rev. 80: in press, 2005.

Buchner E.: Ber. dent. Chem. Gies. 30: 117-124; 1110-1113, 1898.

Chargaff E.: Heraclitean Fire: Sketches from a life before nature. Rockefeller University Press, New York 1978.

Chernavskii D.S., Palamarchuk E.K., Polexhaev A.A., Solyanik G.I., Burlakova E.B.: Mathematical model of periodic processes in membranes with application to cell cycle regulation. BioSystems 9: 187-193, 1977.

Cortassa S., Aon M.A., Iglesias A.A., Lloyd D.: An Introduction to Metabolic and Cellular Engineering. World Scientific, Singapore 2002.
DuPont G., Houart G., Goldbetter A.: From simple to complex $\mathrm{Ca}^{2+}$ oscillations in regulatory mechanisms and theoretical models. In Falke M., Malchow D. (eds), Lecture's in Physics. Understanding Calcium Dynamics Experiments and Theory, pp 131-152.

Edmunds L.N. Jr.: Cellular and Molecular Bases of Biological Clocks. Springer, New York 1988.

Fuentes-Pardo B., Guzman-Gomez A.M., LaraAparicio M., Lopez de Medrano S.: A qualitative model of a motor circadian rhythm. BioSystems 71: 61-69, 2003.

Gilbert D.A.: Ageing, oscillations and efficiency. BioSystems 36: 1-5, 1995.

Gilbert D.A.: The nature of the cell cycle and the control of cell proliferation. BioSystems 5: 197206, 1974.

Gilbert D.A.: The nature of tumour cell proliferation. Nature 311: 160, 1984.

Gonze D., Halloy J., Goldbetter A.: Stochastic models for circadian oscillations: emergence of a biological rhythm. Int. J. Quantum Chem. 98: 228-238, 2004.

Henson M.A.: Modeling the synchronization of yeast respiratory oscillations. J. Theor. Biol. 231: 433-458, 2004.

Kimura H.: Hydrogen sulphide as neuromodulator. Mol. Neurobiol. 26: 13-19, 2002.

Kitano H.: Systems biology: a brief overview. Science 295: 1662-1664, 2002.

Klevecz R.R.: Quantized generation times in mammalian cells as an expression of the cellular clock. Proc. Nat. Acad. Sci. USA. 73: 40124016, 1976.

Klevecz R.R.: A precise circadian clock from chaotic cell cycle oscillations. In Lloyd D., Rossi E.L. (eds): Ultradian rhythms in life processes: an inquiry into fundamental principles of chronobiology and psychobiology. 1993, pp. 41-70.

Klevecz R.R., Bolen J., Forrest G., Murray D.B.: A genomewide oscillation in transcription gates DNA replication and cell cycle. PNAS 101: 1200-1205, 2004.

Lloyd A.L., Lloyd D.: Hypothesis: the central oscillator of the circadian clock is a controlled chaotic oscillator. BioSystems 29: 77-85, 1993.

Lloyd D., Volkov E.I.: Quantized cell cycle times interaction between a relaxation oscillator and ultradian clock pulse. BioSystems 23: 305-310, 1990.

Lloyd D.: Circadian and ultradian clock controlled rhythms in unicellular microorganisms. Adv. Microb. Physiol. 39: 292-339, 1998.

Lloyd D.: Effects of uncoupling of mitochondrial energy conservation on the ultradian clockdriven oscillations in Saccharomyces cerevisiae 
continuous culture. Mitochondrion 3: 139-146, 2003.

Lloyd D., Edwards S.W.: Epigenetic oscillations during the cell cycles of lower eukaryotes are coupled to a clock: life's slow dance to the music of time. In Edmunds L. (ed.): Cell Clocks and Cell Cycles. Plenum Press, New York 1984, pp.27-26.

Lloyd D., Rossi E.R.: Epilogue: the unification hypothesis of chronobiology-psychobiology from molecule to mind. In Lloyd D. and Rossi E.L. (eds.): Ultradian rhythms in life processes: an inquiry into fundamental priciples of chronobiology and psychobiology. 1993, pp. 403-405.

Lloyd D., Lloyd A.L., Olsen L.F.: The cell division cycle: a physiologically plausible dynamic model can exhibit chaotic solutions. BioSystems 27: 17-24, 1992.

Lloyd D., Murray D.B.: Ultradian metronome: timekeeper for orchestration of cellular coherence. Trends Biochem. Soc. 2005.

Lloyd D., Lemar K.M., Murray D.B. et al.: Yeast ultradian clock monitored in continuous cultures and respiratory oscillations evaluated by laser 2-photon microscopy. VII Latin-American Symp. Chronobiology, Tlaxcala, Mexico 2003.

Lloyd D., Lemar K.M., Salgado L.E.J., Gould T.M., Murray D.B.: Respiratory oscillations in yeast: mitochondrial reactive oxygen species, apoptosis and time; an hypothesis. FEMS Yeast Res. 3: 333-339, 2003.

Lloyd D., Poole R.K., Edwards S.W.: The Cell Division Cycle: Temporal Organization and Control of Cellular Growth and Reproduction. Academic Press, London 1982.

Lloyd D., Salgado E.J., Turner M.P., Suller M.T.E., Murray D.B.: Cycles of mitochondrial energization driven by the ultradian clock in a continuous culture of Saccharomyces cerevisiae. Microbiology 148: 3715-3724, 2002.

Mackey M.C., Santary M., Selepora P.: A mitotic oscillator with strange attractor and distributions of cell cycle time. In Othmer H. (ed): Nonlinear Oscillations in Biology and Chemistry, vol 66. Springer, Berlin 1986, pp. 34-45.

Mano Y.: Regulation system of protein synthesis in early embryogenesis in Sea Urchin. Biochem Biophys. Res. Commun. 33: 877-882, 1968.

Murray D.B., Engelen A.A., Keulers M., Kuriyama H., Lloyd D.: $\mathrm{NO}^{+}$, but not $\mathrm{NO}$, inhibits respiratory oscillations in ethanol grown chemostat cultures of Saccharomyces cerevisiae. Biochem. Soc. Trans. 28: 5339, 1998.

Murray D.B., Keulers M., Engelen F., Lloyd D., Kuriyama H.: Involvement of glutathione in the regulation of respiratory oscillations during continuous culture of $S$. cerevisiae. Microbiology, 145: 2739-2745, 1999.

Murray D.B., Klevecz R.R., Lloyd D.: Generation and maintenance of synchrony in Saccharomyces cerevisiae continuous culture. Exp. Cell Res. 287: 10-15, 2003.

Murray D.B., Roller S., Kuriyama H., Lloyd D.: Clock control of ultradian respiratory oscillation found during yeast continuous culture. J. Bacteriol. 183: 7253-7259, 2001.

Nicholson J.K., Holmes E., Lindon J.C., Wilson I.D.: The challenges of modeling mammalian biocomplexity. Nature Biotechnol. 1268-1274, 2004.

Noble D.: Modeling the heart - from genes to cells to the whole organ. Science 295: 1678-1682, 2002.

Noble D.: The heart is already working. Conf. Systems in Biology: Will It Work? Bioch. Soc. Trans., abstract S014, 2005.

Ruoff P., Vinsjevik M., Rensing L.: Temperature compensation in biological oscillators: a challenge for joint experimental and theoretical analysis. Comments Theol. Biol. 5: 361-382, 2000.

Salgado E., Murray D.B., Lloyd D.: Some antidepressant agents $\left(\mathrm{Li}^{+}\right.$, monoamine oxidase type A inhibitors) perturb the ultradian clock in S. cerevisiae. Biol. Rhythm Res. 33: 351-361, 2002.

Satroutdinov A.D., Kuriyama H., Kobayashi H.: Oscillatory metabolism of Saccharomyces cerevisiae in continuous culture. FEMS Microbiol. Lett. 77: 261-267, 1992.

Selkov E.E.: Two alternative self-oscillating stationary states in thiol metabolism - two alternative types of cell division normal and malignant ones (In Russ.). Biofizika 15: 10651073, 1970.

Sohn H.-Y., Murray D.B., Kuriyama H.: Ultradian oscillation of Saccharomyces cerevisiae during aerobic continuous culture: hydrogen sulphide mediates population synchrony. Yeast 16: 1185-1190, 2000.

Strogatz S.H.: From Kuramoto to Crawford: exploring the onset of synchronization in populations of coupled oscillators. Physica. D 143: 1-20, 2000.

Tyson J.J., Chen K.C., Novak B.: Sniffers, buzzers, toggles and blinkers: dynamics of regulatory and signaling pathways in the cell. Curr. Opin. Cell Biol. 15: 221-231, 2003.

von Bertalanffy L.: Problems of Life, Harper, New York 1952.

Weber G.: In Horizons in Biochemistry and Biophysics, vol 2, Addison-Westley Publishing Company 1976, pp. 163-198. 
Weiner N.: The Human Use of Human Beings, Doubleday, New York 1954, pp. 96.

Wolf J., Sohn H.-Y., Heinrich R., Kuriyama H.: Mathematical analysis of a mechanism for autonomous metabolic oscillations in continuous cultures of Saccharomyces cerevisiae. FEBS Lett. 499: 230-234, 2001.
Wolf J., Becker-Weimann S., Heinrich R.: Analysing the robustness of cellular rhythms. Systems Biol. 2005.

Yates F.E.: Fractal applications in biology: scaling time in biochemical networks. Meth. Enzymol. 210: 636-675, 1992. 